Pacific Journal of Mathematic 


\title{
BIORTHOGONAL POLYNOMIALS SUGGESTED BY THE LAGUERRE POLYNOMIALS
}

\author{
Joseph D. E. Konhauser
}

Let $Y_{n}^{c}(x ; k)$ and $Z_{n}^{c}(x ; k), n=0,1, \cdots$, be polynomials of degree $n$ in $x$ and $x^{k}$, respectively, where $x$ is real, $k$ is a positive integer and $c>-1$, such that

$$
\int_{0}^{\infty} x^{c} e^{-x} Y_{n}^{c}(x ; k) x^{k i} d x \text { is }\left\{\begin{array}{l}
0 \text { for } i=0,1, \cdots, n-1 ; \\
\text { not } 0 \text { for } i=n ;
\end{array}\right.
$$

and

$$
\int_{0}^{\infty} x^{c} e^{-x} Z_{n}^{c}(x ; k) x^{i} d x \text { is }\left\{\begin{array}{l}
0 \text { for } i=0,1, \cdots, n-1 ; \\
\text { not } 0 \text { for } i=n .
\end{array}\right.
$$

For $k=1$, conditions (1) and (2) reduce to the orthogonality requirement satisfied by the generalized Laguerre polynomials.

If (1) and (2) hold, then

$$
\int_{0}^{\infty} x^{c} e^{-x} Y_{i}^{c}(x ; k) Z_{j}^{c}(x ; k) d x \text { is }\left\{\begin{array}{l}
0 \text { for } i, j=0,1, \cdots ; i \neq j ; \\
\text { not } 0 \text { for } i=j ;
\end{array}\right.
$$

and conversely.

For both sets of polynomials, we shall establish mixed recurrence relations from which we shall derive differential equations of order $k+1$. From these mixed recurrence relations pure recurrence relations connecting $k+2$ successive polynomials can also be obtained. For $k=1$, the recurrence relations and the differential equations for both of the polynomial sets reduce to those for the generalized Laguerre polynomials.

For $k=2$, the recurrence relations and differential equations are essentially those for polynomials introduced in 1951 by L. Spencer and U. Fano [5] in a paper dealing with the penetration of matter by gamma rays. For the polynomials in $x^{2}$, Spencer and Fano gave formulas, derived mixed recurrence relations, and presented a thirdorder differential equation of the form

$$
A(x) y_{n}^{\prime \prime \prime}+B(x) y_{n}^{\prime \prime}+C(x) y_{n}^{\prime}=\lambda_{n} y_{n},
$$

where $A(x), B(x), C(x)$ are functions of $x$ independent of $n$ and $\lambda_{n}$ is a parameter independent of $x$. In 1958, S. Preiser [2] showed that, apart from real linear transformations, only for the case $k=2$, and only for the weight function $x^{c} e^{-x}, c>-1$, do there exist biorthogonal polynomials in $x^{k}$ satisfying a third-order differential equation of the type (3) and such that the polynomials in $x$ satisfy the adjoint of (3). For each set of polynomials for the case $k=2$, Preiser [2, 3] established 
pure recurrence relations connecting four successive polynomials, as well as several mixed recurrence relations. He also gave a generating function for the polynomials in $x^{2}$.

In 1965, J. D. E. Konhauser [1] considered biorthogonal polynomials in real polynomials $r(x)$ and $s(x)$. He discussed properties which are analogues of properties of orthogonal polynomials. Included were necessary and sufficient conditions for the existence of biorthogonal polynomials, sufficient conditions which ensure the existence of pure recurrence relations, and information on the number and location of the real zeros of the polynomials.

\section{THE POLYNOMIALS IN $x^{k}$}

1. Biorthogonality. The generalized Laguerre polynomials [4], which may be written

$$
L_{n}^{c}(x)=\frac{\Gamma(n+c+1)}{n !} \sum_{j=0}^{n}(-1)^{j}\left(\begin{array}{c}
n \\
j
\end{array}\right) \frac{x^{j}}{\Gamma(j+c+1)}, \quad c>-1,
$$

satisfy the orthogonality condition

$$
\int_{0}^{\infty} x^{c} e^{-x} L_{n}^{c}(x) x^{i} d x=\left\{\begin{array}{l}
0, i=0,1, \cdots, n-1 \\
\neq 0, i=n
\end{array}\right.
$$

Expression (4) and the results of Preiser [2,3] for the case $k=2$ suggest that we investigate the candidacy of the polynomials

$$
Z_{n}^{c}(x ; k)=\frac{\Gamma(k n+c+1)}{n !} \sum_{j=0}^{n}(-1)^{j}\left(\begin{array}{l}
n \\
j
\end{array}\right) \frac{x^{k j}}{\Gamma(k j+c+1)}
$$

as members of one set of a biorthogonal pair.

In (2), we replace $Z_{n}^{c}(x ; k)$ by the right side of (5), then carry out the permissible interchange of summation and integration to obtain

$$
\begin{aligned}
& \frac{\Gamma(k n+c+1)}{n !} \sum_{j=0}^{n}(-1)^{j}\left(\begin{array}{l}
n \\
j
\end{array}\right) \frac{1}{\Gamma(k j+c+1)} \int_{0}^{\infty} e^{-x} x^{k j+c+i} d x \\
& =\frac{\Gamma(k n+c+1)}{n !} \sum_{j=0}^{n}(-1)^{j}\left(\begin{array}{l}
n \\
j
\end{array}\right) \frac{\Gamma(k j+c+i+1)}{\Gamma(k j+c+1)} \\
& =\left.\frac{\Gamma(k n+c+1)}{n !} \sum_{j=0}^{n}(-1)^{j}\left(\begin{array}{c}
n \\
j
\end{array}\right) D^{i} x^{k j+c+i}\right|_{x=1} \\
& =\left.\frac{\Gamma(k n+c+1)}{n !} D^{i} x^{c+i} \sum_{j=0}^{n}(-1)^{j}\left(\begin{array}{l}
n \\
j
\end{array}\right) x^{k j}\right|_{x=1} \\
& =\left.\frac{\Gamma(k n+c+1)}{n !} D^{i} x^{c+i}\left(1-x^{k}\right)^{n}\right|_{x=1},
\end{aligned}
$$


which is zero for $i=0,1, \cdots, n-1$, but is different from zero for $i=n$. Therefore, the polynomials (5) do satisfy orthogonality condition (2).

Before determining the other polynomial set of the biorthogonal pair, we shall establish several relation ships satisfied by the polynomials in $x^{k}$.

2. Mixed recurrence relations. We shall find it convenient to introduce the factorial function defined by

$$
\begin{aligned}
(\alpha)_{0} & =1, \quad \alpha \neq 0 ; \\
(\alpha)_{k}=\prod_{j=1}^{k}(\alpha+j-1) & =\Gamma(\alpha+k) / \Gamma(\alpha), \alpha \neq 0, \quad k \geqq 1 .
\end{aligned}
$$

The first recurrence relation we shall establish is

$$
x D Z_{n}^{c}(x ; k)=n k Z_{n}^{c}(x ; k)-k(k n-k+c+1)_{k} Z_{n-1}^{c}(x ; k),
$$

which is suggested by, and, for $k=1$, reduces to a well-known recurrence relation for the Laguerre polynomials [4].

Considering the difference

$$
n k Z_{n}^{c}(x ; k)-k(k n-k+c+1)_{k} Z_{n-1}^{c}(x ; k),
$$

and using (5), we obtain

$$
\begin{aligned}
& \frac{k \Gamma(k n+c+1)}{(n-1) !}\left[\sum_{j=0}^{n}(-1)^{j}\left(\begin{array}{c}
n \\
j
\end{array}\right) \frac{x^{k j}}{\Gamma(k j+c+1)}\right. \\
& \left.\quad-\sum_{j=0}^{n-1}(-1)^{j}\left(\begin{array}{c}
n-1 \\
j
\end{array}\right) \frac{x^{k j}}{\Gamma(k j+c+1)}\right] \\
& =\frac{k \Gamma(k n+c+1)}{(n-1) !} \sum_{j=0}^{n}(-1)^{j}\left[\left(\begin{array}{c}
n \\
j
\end{array}\right)-\left(\begin{array}{c}
n-1 \\
j
\end{array}\right)\right] \frac{x^{k j}}{\Gamma(k j+c+1)},
\end{aligned}
$$

which may be written

$$
\frac{x \Gamma(k n+c+1)}{n !} \sum_{j=0}^{n}(-1)^{j}\left(\begin{array}{l}
n \\
j
\end{array}\right) \frac{k j x^{k j-1}}{\Gamma(k j+c+1)}=x D Z_{n}^{c}(x ; k),
$$

establishing (6).

Alternatively, we may write (7) as

$$
\begin{aligned}
& \frac{k x^{k} \Gamma(k[n-1]+[c+k]+1)}{(n-1) !} \sum_{j=0}^{n}(-1)^{j}\left(\begin{array}{c}
n-1 \\
j-1
\end{array}\right) \frac{x^{k(j-1)}}{\Gamma(k[j-1]+[c+k]+1)} \\
& =-\frac{k x^{k} \Gamma(k[n-1]+[c+k]+1)}{(n-1) !} \\
& \quad \times \sum_{j=0}^{n-1}(-1)^{j}\left(\begin{array}{c}
n-1 \\
j
\end{array}\right) \frac{x^{k j}}{\Gamma(k j+[c+k]+1)}=-k x^{k} Z_{n-1}^{c+k}(x ; k),
\end{aligned}
$$


which, together with the preceding result, gives the relation

$$
D Z_{n}^{c}(x ; k)=-k x^{k-1} Z_{n-1}^{c+k}(x ; k),
$$

connecting polynomials corresponding to $c$ and $c+k$. For $k=1$, (8) also reduces to a well-known relation for the generalized Laguerre polynomials [4].

3. Differential equation. Still another way of writing the difference (7) is

$$
\frac{k \Gamma(k n+c+1)}{(n-1) !} \sum_{j=0}^{n}(-1)^{j}\left(\begin{array}{c}
n-1 \\
j-1
\end{array}\right) \frac{x^{k j}}{\Gamma(k j+c+1)} \text {, which, in virtue of }
$$

(6), equals $x D Z_{n}^{c}(x ; k)$. Multiplying by $x^{c}$ and taking the $k$ th derivative, we obtain

$$
\begin{aligned}
\frac{k \Gamma(k n+c+1)}{(n-1) !} \sum_{j=0}^{n}(-1)^{j}\left(\begin{array}{c}
n-1 \\
j-1
\end{array}\right) \frac{x^{k(j-1)+c}}{\Gamma(k j-k+c+1)} \\
=-k(k n-k+c+1)_{k} x^{c} \frac{\Gamma(k n-k+c+1)}{(n-1) !} \\
\quad \times \sum_{j=0}^{n-1}(-1)^{j}\left(\begin{array}{c}
n-1 \\
j
\end{array}\right) \frac{x^{k j}}{\Gamma(k j+c+1)} \\
=-k(k n-k+c+1)_{k} x^{c} Z_{n-1}^{c}(x ; k) .
\end{aligned}
$$

Therefore,

$$
D^{k}\left[x^{c+1} D Z_{n}^{c}(x ; k)\right]=-k x^{c}(k n-k+c+1)_{k} Z_{n-1}^{c}(x ; k) .
$$

Combining (6) and (9), eliminating $Z_{n-1}^{c}(x ; k)$, we obtain the differential equation of order $k+1$

$$
D^{k}\left[x^{c+1} D Z_{n}^{c}(x ; k)\right]=x^{c+1} D Z_{n}^{c}(x ; k)-n k x^{c} Z_{n}^{c}(x ; k)
$$

for the polynomials in $x^{k}$.

It is not difficult to verify directly that the polynomials in (5) do satisfy (10). For $k=1,(10)$ reduces to the differential equation for the generalized Laguerre polynomials [4]. For $k=2$, (10) reduces to the differential equation which was given by Spencer and Fano and which was derived by Preiser [2, 3] by applying necessary conditions for the existence of biorthogonal polynomial solutions to the coefficients $A(x), B(x), C(x)$ in (3).

For $k>2$, it is not known if the polynomials $Z_{n}^{c}(x ; k)$ satisfy a differential equation of the form

$$
\sum_{j=1}^{m} A_{j}(x) y_{n}^{(j)}=\lambda_{n} y_{n}, m<k+1 .
$$

Preiser $[2,3]$ has shown the answer to be negative for $k=2$. 
4. Pure recurrence relation. Applying Leibniz's rule for the $k$ th derivative of a product to the left side of (9), we get

$$
\sum_{i=0}^{k}\left(\begin{array}{c}
k \\
i
\end{array}\right)\left[D^{k-i} x^{c+1}\right]\left[D^{i+1} Z_{n}^{c}(x ; k)\right]=-k x^{c}(k n-k+c+1)_{k} Z_{n-1}^{c}(x ; k) .
$$

The left side is a sum of derivatives of $Z_{n}^{c}(x ; k)$ from the first through the $k+1$ st. Elimination of the derivatives by repeated use of (6) leads to a pure recurrence relation connecting $k+2$ successive polynomials. An alternate method for obtaining the pure recurrence relation is given in [1].

We now turn to the polynomials in $x$ which satisfy orthogonality condition (1).

\section{THE POLYNOMIALS IN $x$}

1. A suggested recurrence relation. We seek coefficients $a_{n, j}$ such that the polynomials

$$
\sum_{j=0}^{n} a_{n, j} x^{n-j}
$$

satisfy orthogonality condition (1). Taking $n=0,1,2,3$ and using a method of undetermined coefficients, we obtain, each to within an arbitrary multiplicative constant, the first four polynomials

$$
\begin{aligned}
& 1, \\
& x-(c+1), \\
& x^{2}-(k+2 c+3) x+(c+1)(k+c+1), \\
& x^{3}-(3 k+3 c+6) x^{2}+[(2 k+c+2)(k+2 c+3) \\
& \quad+(c+1)(k+c+1)] x-(c+1)(k+c+1)(2 k+c+1) .
\end{aligned}
$$

For $k=1$, the polynomials reduce to the generalized Laguerre polynomials taken to be monic, so in a sense these polynomials, as well as the polynomials in $x^{k}$, may be considered generalizations of the Laguerre polynomials.

The pattern of coefficients suggests the following difference equation for the coefficients

$$
a_{n, j}=-[(k+1) n-j+(-k+c+1)] a_{n-1, j-1}+a_{n-1, j^{\prime}}
$$

where $a_{n, 0}=1$ for all $n$ and $a_{i, j}=0$ if $i<j$. We shall use (13) as the basis of a conjecture for a recurrence relation for the polynomials in $x$. Then we shall use the recurrence relation to show that the polynomials satisfying it satisfy (1). By uniqueness, established in [1], the polynomials we seek are the polynomials which satisfy the recurrence relation. 
First, we shall sacrifice the monic property of the polynomials by modifying the difference equation (13) to

$$
k n b_{n, j}=\left[k n+j+(-k+c+1) b_{n-1, j}-b_{n-1, j-1}\right.
$$

where $b_{0,0}=1, b_{i, j}=0$ if $i<j, b_{i, \ldots 1}=0$ for all $i$, so that the polynomials in $x$ are given by

$$
Y_{n}^{c}(x ; k)=\sum_{j=0}^{n} b_{n, j} x^{j} .
$$

A result of the modification is that, for $k=1$, (14) is a recurrence relation for the coefficients of the generalized Laguerre polynomials.

Substituting for $b_{n, j}$ in (15), we get

$$
k n Y_{n}^{c}(x ; k)=\sum_{j=0}^{n}[k n+j+(-k+c+1)] b_{n-1, j} x^{j}-\sum_{j=0}^{n} b_{n-1, j-1} x^{j} .
$$

Replacing $n$ by $n+1$, and noting that $b_{n, n+1}=0$, we have

$$
\begin{aligned}
k(n+1) Y_{n+1}^{c}(x ; k) & =\sum_{j=0}^{n+1}[k n+j+c+1] b_{n, j} x^{j}-\sum_{j=0}^{n+1} b_{n, j-1} x^{j} \\
& =(k n+c+1) Y_{n}^{c}(x ; k)+\sum_{j=0}^{n} j b_{n, j} x^{j}-\sum_{j=0}^{n} b_{n, j} x^{j+1} .
\end{aligned}
$$

The first sum on the right side is $x D Y_{n}^{c}(x ; k)$ and the second is $x Y_{n}^{c}(x ; k)$; therefore, a suggested recurrence relation for the polynomials in $x$ is

$$
k(n+1) Y_{n+1}^{c}(x ; k)=x D Y_{n}^{c}(x ; k)+(k n+c+1-x) Y_{n}^{c}(x ; k) .
$$

2. Biorthogonality. To establish that the polynomials in $x$ satisfying (16) comprise the other set of the biorthogonal pair, we must show that (1) is satisfied. We proceed by induction.

For $n=0$, the integral in (1) has the nonzero value $\Gamma(c+1)$ for the only permissible value of $i$, namely $i=0$.

For $n=1$, we must verify that the integral in (1) is zero for $i=0$ and nonzero for $i=1$. For $i=0$, we have

$$
\begin{aligned}
\int_{0}^{\infty} x^{c} e^{-x} Y_{1}^{c}(x ; k) d x & =\int_{0}^{\infty} x^{c} e^{-x} k^{-1}(c+1-x) d x \\
& =k^{-1}[(c+1) \Gamma(c+1)-\Gamma(c+2)]=0,
\end{aligned}
$$

where $Y_{1}^{c}(x ; k)=k^{-1}(c+1-x)$ was obtained from (16) for $n=0$.

For $i=1$, we have

$$
\begin{aligned}
\int_{0}^{\infty} x^{c} e^{-x} x^{k} Y_{1}^{c}(x ; k) d x & =\int_{0}^{\infty} x^{c+k} e^{-x} k^{-1}(c+1-x) d x \\
& =k^{-1}[(c+1) \Gamma(c+k+1)-\Gamma(c+k+2)] \\
& =-\Gamma(c+k+1) \neq 0 .
\end{aligned}
$$


Continuing the induction argument, we assume that the polynomials $Y_{i}^{c}(x ; k), i=0,1, \cdots, n$, obtained by repeated application of (16), satisfy orthogonality relation (1). To complete the induction argument, we must show that

$$
\int_{0}^{\infty} x^{c} e^{-x} Y_{n+1}^{c}(x ; k) x^{i k} d x=\left\{\begin{aligned}
0, i & =0,1, \cdots, n \\
\neq 0, i & =n+1
\end{aligned}\right.
$$

Substituting for $Y_{n+1}^{c}(x ; k)$, as given by $(16)$, we obtain

$$
\begin{aligned}
& k^{-1}(n+1)^{-1} \int_{0}^{\infty} x^{c+i k+1} e^{-x} D Y_{n}^{c}(x ; k) d x \\
& \quad+k^{-1}(n+1)^{-1} \int_{0}^{\infty}(k n+c+1-x) x^{c+i k} e^{-x} Y_{n}^{c}(x ; k) d x .
\end{aligned}
$$

If we integrate the first integral by parts the sum becomes

$$
\begin{aligned}
& k^{-1}(n+1)^{-1} \int_{0}^{\infty}\left[x^{c+i k+1}-(c+i k+1) x^{c+i k}\right] e^{-x} Y_{n}^{c}(x ; k) d x \\
& \quad+k^{-1}(n+1)^{-1} \int_{0}^{\infty}(k n+c+1-x) x^{c+i k} e^{-x} Y_{n}^{c}(x ; k) d x,
\end{aligned}
$$

which may be written

$$
(n+1)^{-1} \int_{0}^{\infty} x^{c+i k} e^{-x}(n-i) Y_{n}^{c}(x ; k) d x .
$$

The integral in (18) is zero for $i=n$ since the integrand is zero. By hypothesis, $Y_{n}^{c}(x ; k)$ is orthogonal to $x^{i k}, 0 \leqq i<n$; therefore, for $i<n$, the integral in (18) is zero.

For $i=n+1$, the integral has the value

$$
-(n+1)^{-1} \int_{0}^{\infty} x^{c+k n+k} e^{-x} Y_{n}^{c}(x ; k) d x=(-1)^{n+1} \Gamma(c+k n+k+1),
$$

which is different from zero, the right side having been obtained by $n$ applications of (16) each followed by an integration by parts.

3. Expression for $Y_{n}^{c}(x ; k)$. Preiser $[2,3]$ obtained a closed form for the polynomials in $x$ for the case $k=2$ by applying Cauchy's Theorem to the integral-form solution of

$$
\begin{aligned}
x D^{3} Y_{n}(x ; 2) & +(1+c-3 x) D^{2} Y_{n}(x ; 2) \\
& +2(x-1-c) D Y_{n}(x ; 2)=2 n Y_{n}(x ; 2) .
\end{aligned}
$$

A closed form for the polynomials is desirable but is not essential, since certain properties of the polynomials can be established without one. By a method similar to that of Preiser, we shall find polynomials solutions of (19) in integral form, conjecture the form of the integral 
for the general case, show that the polynomials so obtained satisfy (16), and then, using the integral form, establish recurrence relations which will be used to derive a differential equation for the polynomials.

Equation (19) may be written

$$
x\left(y^{\prime \prime \prime}-3 y^{\prime \prime}+2 y^{\prime}\right)+\left[(1+c) y^{\prime \prime}-2(1+c) y^{\prime}-2 n y\right]=0 .
$$

We assume a solution of the form

$$
y=\int_{o} e^{-x t} \phi(t) d t,
$$

where the function $\phi(t)$ and the contour $C$ are to be determined.

Differentiating successively and substituting into (20), we get

$$
\begin{aligned}
& -x \int_{\sigma}\left(t^{3}+3 t^{2}+2 t\right) e^{-x t} \phi(t) d t \\
& \quad+\int_{\sigma}\left[(1+c) t^{2}+2(1+c) t-2 n\right] e^{-x t} \phi(t) d t=0 .
\end{aligned}
$$

Integrating the first integral by parts, we obtain

$$
\begin{aligned}
0= & \left.\left(t^{3}+3 t^{2}+2 t\right) \phi(t) e^{-x t}\right|_{o}-\int_{o} e^{-x t}\left[\left(3 t^{2}+6 t+2\right) \phi(t)\right. \\
& \left.+\left(t^{3}+3 t^{2}+2 t\right) \phi^{\prime}(t)\right] d t+\int_{o}\left[(1+c) t^{2}+2(1+c) t-2 n\right] e^{-x t} \phi(t) d t
\end{aligned}
$$

We shall choose $\phi(t)$ so that

$$
\begin{aligned}
& {\left[(1+c) t^{2}+2(1+c) t-2 n-3 t^{2}-6 t-2\right] \phi(t)} \\
& \quad-\left(t^{3}+3 t^{2}+2 t\right) \phi^{\prime}(t)=0
\end{aligned}
$$

and the contour $C$ such that

$$
\left.\left(t^{3}+3 t^{2}+2 t\right) \phi(t) e^{-x t}\right|_{\sigma}=0
$$

From (22), we have

$$
\frac{\phi^{\prime}(t)}{\phi(t)}=-\frac{n+1}{t}+\frac{c+2 n}{t+1}-\frac{n+1}{t+2},
$$

whence $\phi(t)=K(t+1)^{c+2 n} / t^{n+1}(t+2)^{n+1}$, where $K$ is an arbitrary constant which we shall take equal to $k / 2 \pi i$.

Substituting into (23), we require the contour $C$ to be such that

$$
\left.\frac{k}{2 \pi i} \frac{(t+1)^{c+2 n+1}}{t^{n}(t+2)^{n}}\right|_{o}=0 \text {. }
$$

If we take $C$ to be a closed contour encircling $t=0$, but not $t=-1$ or $t=-2$, then (23) holds and we have 


$$
y=\frac{k}{2 \pi i} \int_{0} \frac{e^{-x t}(t+1)^{c+2 n}}{t^{n+1}(t+2)^{n+1}} d t .
$$

On the basis of this integral, we conjecture that the polynomials $Y_{n}^{c}(x ; k)$ are given by

$$
\phi_{n}(x)=\frac{k}{2 \pi i} \int_{0} \frac{e^{-x t}(t+1)^{c+k n}}{\left[(t+1)^{k}-1\right]^{n+1}} d t .
$$

In view of the uniqueness established in [1], it suffices to show that the polynomials (24) satisfy recurrence relation (16).

We have

$$
x D \phi_{n}(x)-x \phi_{n}(x)=-\frac{k}{2 \pi i} \int_{\sigma} \frac{e^{-x t}(t+1)^{c+k n+1}}{\left[(t+1)^{k}-1\right]^{n+1}} d t .
$$

Integrating by parts, and applying (24), the right side of (25) becomes

$$
-(c+k n+1) \phi_{n}(x)+k(n+1) \dot{\phi}_{n+1}(x) .
$$

Therefore,

$$
x D \phi_{n}(x)-x \phi_{n}(x)=-(c+k n+1) \phi_{n}(x)+k(n+1) \phi_{n+1}(x),
$$

which is (16) with $\phi_{n}(x)$ in place of $Y_{n}^{c}(x ; k)$.

In summary, we have

$$
Y_{n}^{c}(x ; k)=\frac{k}{2 \pi i} \int_{o} \frac{e^{-x t}(t+1)^{c+k n}}{\left[(t+1)^{k}-1\right]^{n+1}} d t .
$$

Applying Cauchy's theorem to (26), we obtain the following representation for the polynomials in $x$ :

$$
Y_{n}^{c}(x ; k)=\left.\frac{k}{n !} \frac{\partial^{n}}{\partial t^{n}}\left[\frac{e^{-x t}(t+1)^{c+k n}}{\left(t^{k-1}+k t^{k-2}+\cdots+k\right)^{n+1}}\right]\right|_{t=0} .
$$

We shall use (26) to obtain mixed recurrence relations for the polynomials $Y_{n}^{c}(x ; k)$.

4. Mixed recurrence relations, Observing that

$$
(t+1)^{c+k n}=(t+1)^{c+k n+k}-(t+1)^{c+k n}\left[(t+1)^{k}-1\right],
$$

we write (26) as

$$
\begin{aligned}
Y_{n}^{c}(x ; k) & =\frac{k}{2 \pi i} \int_{0} \frac{e^{-x t}(t+1)^{c+k n+k}}{\left[(t+1)^{k}-1\right]^{n+1}} d t-\frac{k}{2 \pi i} \int_{o} \frac{e^{-x t}(t+1)^{c+k(n-1)+k}}{\left[(t+1)^{k}-1\right]^{n}} d t \\
& =Y_{n}^{c+k}(x ; k)-Y_{n-1}^{c+k}(x ; k)
\end{aligned}
$$

which, for $k=1$, reduces to a well-known relation for the Laguerre 
polynomials [4].

Next, we shall derive a recurrence relation which, together with (16), leads to a differential equation satisfied by the polynomials in $x$. We have

$$
Y_{n+1}^{c}(x ; k)=\frac{k}{2 \pi i} \int_{o} \frac{e^{-x t}(t+1)^{c+k n+k}}{\left[(t+1)^{k}-1\right]^{n+2}} d t .
$$

Operating on $Y_{n+1}^{c}(x ; k)$ with $(D-1)^{k}-(-1)^{k}$, we obtain

$$
\frac{k(-1)^{k}}{2 \pi i} \int_{0} \frac{e^{-x t}(t+1)^{c+k n+k}}{\left[(t+1)^{k}-1\right]^{n+1}} d t,
$$

which is precisely the result of operating on $Y_{n}^{c}(x ; k)$ with $(D-1)^{k}$. Therefore,

$$
\left[(D-1)^{k}-(-1)^{k}\right] Y_{n+1}^{c}(x ; k)=(D-1)^{k} Y_{n}^{c}(x ; k) .
$$

5. Differential equation. We shall now derive a differential equation satisfied by the polynomials in $x$. The equation turns out to be of order $k+1$, which is the order of the differential equation (10) satisfied by the polynomials in $x^{k}$. The form of the differential equation satisfied by the polynomials in $x$ is simpler than (10), the coefficients being no higher than first degree in $x$.

In deriving the final form of the differential equation we shall apply the following easily established

Lemma. If $y$ is a differentiable function of $x$ possessing $k+1$ derivatives, then

$$
(D-1)^{k} x(D-1) y=\left[x(D-1)^{k+1}+k(D-1)^{k}\right] y .
$$

From (16)

$$
k(n+1) Y_{n+1}^{c}(x ; k)=[x(D-1)+c+k n+1] Y_{n}^{c}(x ; k) .
$$

Substituting into (27), we get

$$
\begin{aligned}
& {\left[(D-1)^{k}-(-1)^{k}\right][x(D-1)+c+k n+1] Y_{n}^{c}(x ; k)} \\
& \quad=k(n+1)(D-1)^{k} Y_{n}^{c}(x ; k),
\end{aligned}
$$

which may be written

$$
\begin{aligned}
& {\left[\left[(D-1)^{k}-(-1)^{k}\right][x(D-1)+c+1]-k n(-1)^{k}\right] Y_{n}^{c}(x ; k)} \\
& \quad=k(D-1)^{k} Y_{n}^{c}(x ; k) .
\end{aligned}
$$

Applying the identity (28) to (29), we obtain, after some rearrangement of terms, the differential equation 
(30)

$$
\left[[x(D-1)+c+1]\left[(D-1)^{k}-(-1)^{k}\right]-(-1)^{k} k n\right] Y_{n}^{c}(x ; k)=0,
$$

which may be written in the form

$$
[x \Psi(D)+\Omega(D)] y=0 .
$$

Equations of the form (31) are solvable by the method used to solve (20), the solutions being of the form (26).

For $k=2$, (30) reduces to the equation for the polynomials in $x$ of Spencer and Fano [5, 2, 3].

For $k=1$, (30) reduces to the equation for the generalized Laguerre polynomials [4], as did equation (10).

6. Pure recurrence relation. By eliminating the derivatives between (16) and (29), using (28), one can obtain a pure recurrence relation connecting $k+2$ successive polynomials in $x$. An alternate method for obtaining the pure recurrence relation is given in [1].

\section{THE INTEGRALS $J_{n, n}$}

We conclude our discussion of the biorthogonal polynomials $Z_{n}^{c}(x ; k)$ and $Y_{n}^{c}(x ; k)$ with an evaluation of the integral

$$
J_{n, n}=\int_{0}^{\infty} x^{c} e^{-x} Y_{n}^{c}(x ; k) Z_{n}^{c}(x ; k) d x .
$$

First, we shall show that $b_{n, n}=(-1)^{n} / k^{n} n !, n=0,1,2, \cdots$. We proceed by induction. For $n=0$, we have $b_{0,0}=1$. For $n=1$, from (14), we get $b_{1,1}=-1 / k$. We assume that $b_{n-1, n-1}=(-1)^{n-1} / k^{n-1}(n-1)$ !. Taking $j=n$ in (14), and noting that $b_{n-1, n}=0$, we get

$$
b_{n, n}=-b_{n-1,{ }_{n-1}} / k n=(-1)^{n} / k^{n} n !,
$$

completing the induction argument.

In virtue of the orthogonality of $x^{j}$ and $Z_{n}^{c}(x ; k)$ for $j<n$, we have

$$
J_{n, n}=\int_{0}^{\infty} x^{c} e^{-x} Z_{n}^{c}(x ; k) \sum_{j=0}^{n} b_{n, j} x^{j} d x=\int_{0}^{\infty} x^{c} e^{-x} Z_{n}^{c}(x ; k) b_{n, n} x^{n} d x .
$$

Substituting for $b_{n, n}$, and proceeding as in the establishment of the biorthogonality property of the polynomials in $x^{k}$, we obtain

$$
\begin{aligned}
J_{n, n} & =\left.\frac{(-1)^{n}}{k^{n} n !} \frac{\Gamma(k n+c+1)}{n !} D^{n} x^{c+n}\left(1-x^{k}\right)^{n}\right|_{x=1} \\
& =\frac{\Gamma(k n+c+1)}{n !},
\end{aligned}
$$

which, for $k=1$, is the value of the corresponding integral for the 
generalized Laguerre polynomials [4].

\section{BIBLIOGRAPHY}

1. J. D. E. Konhauser, Some Properties of Biorthogonal Polynomials, Journal of Mathematical Analysis and Applications 11 (1965), 242-260.

2. S. Preiser, An Investigation of Biorthogonal Polynomials Derivable from Ordinary Differential Equations of the Third Order, Ph. D. Thesis, New York University, 1958. 3. — An investigation of biorthogonal polynomials derivable from ordinary differential equations of the third order, Journal of Mathematical Analysis and Applications 4 (1962), 38-64.

4. E. Rainville, Special Functions, Macmillan, 1960.

5. L. Spencer and U. Fano, Penetration and diffusion of X-rays. Calculation of spatial distributions by polynomial expansion, Journal of Research, National Bureau of Standards 46 (1951), 446-461.

Received September 9, 1965.

UNIVERSITY OF MINNESOTA 


\section{PACIFIC JOURNAL OF MATHEMATICS}

\section{EDITORS}

H. SAMELSON

Stanford University

Stanford, California

J. P. JANS

University of Washington

Seattle, Washington 98105

\section{J. DugunduI}

University of Southern California Los Angeles, California 90007

RICHARD ARENS

University of California

Los Angeles, California 90024

\section{ASSOCIATE EDITORS}
E. F. BECKENBACH
B. H. NEUMANN
F. WOLF
K. YoSIDA

\section{SUPPORTING INSTITUTIONS}

\author{
UNIVERSITY OF BRITISH COLUMRIA \\ CALIFORNIA INSTITUTE OF TECHNOLOGY \\ UNIVERSITY OF CALIFORNIA \\ MONTANA STATE UNIVERSITY \\ UNIVERSITY OF NEVADA \\ NEW MEXICO STATE UNIVERSITY \\ OREGON STATE UNIVERSITY \\ UNIVERSITY OF OREGON \\ OSAKA UNIVERSITY \\ UNIVERSITY OF SOUTHERN CALIFORNIA
}

\author{
STANFORD UNIVERSITY \\ UNIVERSITY OF TOKYO \\ UNIVERSITY OF UTAH \\ WASHINGTON STATE UNIVERSITY \\ UNIVERSITY OF WASHINGTON \\ AMERICAN MATHEMATICAL SOCIETY \\ CHEVRON RESEARCH CORPORATION \\ TRW SYSTEMS \\ NAVAL ORDNANCE TEST STATION
}

Mathematical papers intended for publication in the Pacific Journal of Mathematics should be typewritten (double spaced). The first paragraph or two must be capable of being used separately as a synopsis of the entire paper. It should not contain references to the bibliography. Manuscripts may be sent to any one of the four editors. All other communications to the editors should be addressed to the managing editor, Richard Arens at the University of California, Los Angeles, California 90024 .

50 reprints per author of each article are furnished free of charge; additional copies may be obtained at cost in multiples of 50 .

The Pacific Journal of Mathematics is published monthly. Effective with Volume 16 the price per volume (3 numbers) is $\$ 8.00$; single issues, $\$ 3.00$. Special price for current issues to individual faculty members of supporting institutions and to individual members of the American Mathematical Society: $\$ 4.00$ per volume; single issues $\$ 1.50$. Back numbers are available.

Subscriptions, orders for back numbers, and changes of address should be sent to Pacific Journal of Mathematics, 103 Highland Boulevard, Berkeley 8, California.

Printed at Kokusai Bunken Insatsusha (International Academic Printing Co., Ltd.), No. 6, 2-chome, Fujimi-cho, Chiyoda-ku, Tokyo, Japan.

\section{PUBLISHED BY PACIFIC JOURNAL OF MATHEMATICS, A NON-PROFIT CORPORATION}

The Supporting Institutions listed above contribute to the cost of publication of this Journal, but they are not owners or publishers and have no responsibility for its content or policies. 


\section{Pacific Journal of Mathematics}

\section{Vol. 21, No. 2 December, 1967}

Arne P. Baartz, The measure algebra of a locally compact semigroup ..... 199

Robert F. Brown, On maps with identical fixed point sets............. 215

C. Buttin, Existence of a homotopy operator for Spencer's sequence in the analytic case ..................................... 219

Henry Werner Davis, An elementary proof that Haar measurable almost periodic functions are continuous ........................ 241

Zeev Ditzian, On asymptotic estimates for kernels of convolution transforms ...................................... 249

Robert E. Edwards, Boundedness principles and Fourier theory ......... 255

John A. Hildebrant, On compact unithetic semigroups ............... 265

Marinus A. Kaashoek and David Clark Lay, On operators whose Fredholm set is the complex plane ............................ 275

Sadao Kató, Canonical domains in several complex variables ........... 279

David Clifford Kay, The ptolemaic inequality in Hilbert geometries.... . . . 293

Joseph D. E. Konhauser, Biorthogonal polynomials suggested by the Laguerre polynomials ............................. 303

Kevin Mor McCrimmon, Macdonald's theorem with inverses .......... 315

Harry Eldon Pickett, Homomorphisms and subalgebras of multialgebras .................................... 327

Richard Dennis Sinkhorn and Paul Joseph Knopp, Concerning nonnegative matrices and doubly stochastic matrices ..............

Erling Stormer, On anti-automorphisms of von Neumann algebras ...

Miyuki Yamada, Regular semi-groups whose idempotents satisfy permutation identities .......................... 